\title{
Analytical Investigation of Active Compound Contents of Panax ginseng C.A. Meyer and Ginkgo biloba L. Supplements Fortified with Apitherapy Products
}

\author{
Ismail Emir Akyildiz ${ }^{(1,2, *}$, Sinem Raday ${ }^{(12}$, Ozge Erdem ${ }^{(2)}$, Sezer Acar ${ }^{(\mathbb{D} 2}$, \\ Ilknur Coskun ${ }^{2}$, Emel Damarli ${ }^{2}$
}

\author{
${ }^{1}$ Department of Chemistry, Faculty of Science, Marmara University, 34722, Istanbul, Turkey \\ ${ }^{2}$ Altiparmak Food Co. R\&D Center, 34782, Istanbul, Turkey
}

\begin{abstract}
Extracts of therapeutic plants of nature, such as Ginkgo biloba L. and Panax ginseng C.A. Meyer (P.ginseng) are highly requested. Recently admixtures of these extracts with apitherapy products are also available. In this study, as research materials, P.ginseng, and Ginkgo biloba L. supplements in the form of tablets, paste, and liquid extracts prepared as a mixture with bee products were compared in terms of their ginsenosides, phenolics, terpene lactones, and antioxidant capacities. Within this study, it was aimed to clarify the active ingredients P.ginseng extracts, Ginkgo biloba L. extracts, and their mixtures with bee products by developing advanced and novel analytical methods. Additionally, it was purposed to unveil the contribution of bee products to phytotherapy supplements of P.ginseng and Ginkgo biloba L. on the basis of secondary metabolites. Ultrasound-assisted hydroalcoholic extraction was applied at sample preparation and supernatants were analyzed at UPLC-MS/MS and UV-Visible spectrophotometer. The antioxidant activities were determined using the 2,2diphenyl-1-picrylhydrazyl (DPPH) method. As a result, total ginsenoside contents of the products per serving were ranged between $9.7 \mathrm{mg}$ to $150.2 \mathrm{mg}$. Terpene lactones of the ginkgo products were ranged between $0.1 \mathrm{mg}$ to $9.5 \mathrm{mg}$ per serving. The antioxidant activities of all products were determined between $13 \%$ and $92 \%$. Quercetin, rutin hydrate, CAPE, kaempferol, galangin, chrysin, gallic acid, pinocembrin, and isorhamnetin were found as the predominant phenolics. Due to their higher antioxidant capacities, superior phenolic concentrations, and diversities, it was enlightened that apitherapy products can augment the phytotherapy efficacies of P.ginseng and Ginkgo biloba L. supplements.
\end{abstract}

\section{ARTICLE HISTORY}

Received: Nov. 27, 2021

Revised: Apr. 21, 2021

Accepted: May 06, 2021

\section{KEYWORDS}

Panax.ginseng,

Ginkgo biloba L.,

UPLC-MS,

Apitherapy,

Phenolics.

\section{INTRODUCTION}

With the development of modern medicines and revisions to the methods of treatment, phytotherapy which has been thrown into the background for a while has become popular again due to the difficulties in the treatment of chronic diseases and increased side effects from some modern therapies. Plant extracts of therapeutic nature, such as Panax ginseng C.A. Meyer and Ginkgo biloba L., dating back to ancient times, and mixtures containing these extracts are being 
requested by the consumers. In recent years, the mixture forms of these extracts produced from such plants with alternative apitherapy products are available at pharmacies and herbalists and these preparations attract great attention.

Ginseng is a slow-growing, perennial, deciduous herb that belongs to the Araliaceae family and the Panax genus. The most known and important cultivated species are Panax ginseng C.A. Meyer (Asian ginseng) and Panax quinquefolius L. (American ginseng). P. ginseng has been the main source of ginseng root used at traditional medicines and it is classified into three types depending on drying conditions (Popovich et al., 2012). Panax quinquefolius L. is native to North America. It is 'cool' and is traditionally used for decreasing the 'internal heat' as opposed to $P$. ginseng which has heating effects (Gui \& Ryu, 2014; Popovich et al., 2012). Carbohydrates such as polysaccharides, starch and cellulose are the major components of P.ginseng (60-70\%) (Lee et al., 2015). P.ginseng also contains antioxidative compounds, polyacetylenes, and gomisins, which protect the liver and peptides that behave likewise to insulin (Qi et al., 2011; Shin et al., 2015). In traditional Chinese medicine, ginseng is commonly mentioned as an "adaptogen," a substance that can aid an organism in getting over various types of stress and restore homeostasis. Immunomodulating and bio-modulating action, beneficial effects within the hematopoietic, cardiovascular, endocrine, immune, and central nervous systems are among the most important features of ginseng in clinical trials and laboratory (Kim, 2018; Kim et al., 2017; Kim et al., 2018). Different ginsenosides are responsible for these health-promoting effects, therefore it is important to determine the composition of various ginsenosides in commercial ginseng products. Ginsenosides are the major secondary metabolites found in roots more than other parts of the ginseng plant, which are a class of triterpene saponins (Kim et al., 2017). In both P. ginseng and Panax quinquefolius L., ginsenosides Re, Ro, Rg1, Rb1, Rb2, Rc and Rd are present in different amounts, while ginsenoside $\mathrm{Rf}$ can only be identified in $P$. ginseng (Dou et al., 1998) and its products are commercially marketed as dietary supplements with the forms of dried root powders, liquid extracts, teas and formulated into tablets, capsules, etc. The recommended dose of $P$. ginseng standardized extract containing 1.5 to $7 \%$ ginsenosides is $100-300 \mathrm{mg}$ per day or 0.5 to $2 \mathrm{~g}$ per day of the root extract (Court et al., 1996). Through modern analytical technology, at least 289 ginsenosides were reported from 11 species of genus Panax. The most general subtype of ginsenosides (126 reported compounds) has C-17 side chains. In addition, 66 20(S)-/ 20(R)protopanaxadiol, 50 20(S)- or 20(R)-protopanaxatriol, 19 oleanolic acid, 15 ocotillo, and 13 miscellaneous saponin compounds have been reported (Kim, 2018; Yang et al., 2014).

In literature, Soxhlet extraction, ultrasound-assisted extraction, alkaline hydrolysis, refluxing methods have been typically used to extract ginsenosides from roots of $P$. ginseng with hydroalcoholic solvents as the typical organic modifiers (Hong et al., 2009; Shin et al., 2001a, 2001b). High-performance liquid chromatography (HPLC) has been generally used to determine the ginsenosides with refractive index (RI), ultraviolet (UV) detections and mass spectrometers (MS) (Chan et al., 2000; Li et al., 2005; Xia et al., 2018; Yu et al., 2021).

The other widely consumed herbal nutritional product is Ginkgo biloba L. which is one of the oldest species (family Ginkgoaceae). It is native to China and also grows throughout Japan, Korea, Europe, and the United States. Ginkgo trees reach a height of $20-35 \mathrm{~m}$ and can live for as long as 1000 years (Jacobs \& Browner, 2000). The medicinal properties of Ginkgo biloba L. can be traced back to 5.000 years in China. The healer Chen Noung described the medicinal properties of the Ginkgo biloba L. in the first known pharmacopeia (Chang \& Chang, 1997). Medicinal extracts are made from dried leaves and have been used for various purposes (DeFeudis, 1998). Uses of Ginkgo biloba L. extracts include the treatment and/or prevention of age-related physical and mental impairments, Alzheimer's, cardiovascular and bronchial disease (Diamond et al., 2000; Le Bars et al., 2002; Pietri et al., 1997). Such pharmacological 
activities are attributed to the synergistic activity of the flavonoids and the terpene trilactones (Smith \& Luo, 2004; Stiker et al., 2000; van Beek, 2002). The flavonoids are composed of a large group of polyphenols and contain flavonol glycosides, flavones, flavan-3-ols, acylated flavonol glycosides and proanthocyanidins. Among these molecules, the flavonol glycosides are the most abundant group. Furthermore, numerous flavonol glycosides are sometimes found as their aglycones such as kaempferol, quercetin and isorhamnetin that are usually present in the leaves with small amounts (van Beek, 2002). The ginkgo terpene trilactones: bilobalide (sesquiterpene) and ginkgolides (diterpene); ginkgolide $\mathrm{B}$, ginkgolide $\mathrm{A}$, ginkgolide $\mathrm{J}$ and ginkgolide $\mathrm{C}$ are unique to Ginkgo biloba $\mathrm{L}$. and they show broad spectrum of pharmacological activities (de Jager et al., 2006). Commercial Ginkgo biloba L. products are usually commercialized as mixtures, based on the content of terpene lactones and flavonol glycosides (van Beek \& Montoro, 2009). A standardized leaf extract contains 22-27\% of flavone glycosides and $5-7 \%$ of terpene lactones. Ginkgolide A, C, B with $2.8-3.4 \%$ and bilobalide with $2.6-3.2 \%$ along with less than 5 ppm ginkgolic acid are expected to be observed at standardized leaf extract (Kiefer, 2004). The most considered standardized leaf extract in studies is EGb761 (Mahadevan \& Park, 2008). The standard dose of EGb761 is $120 \mathrm{mg}(\sim 1.7$ $\mathrm{mg} / \mathrm{kg}$ ) once or twice daily for clinical puposed usage; hence, a standard dose will contain $\sim 3-$ $4 \mathrm{mg}$ ginkgolides A, B, and C, 3-4 mg bilobalide, and $29 \mathrm{mg}$ flavonoids (Nash \& Shah, 2015). Quality control of Ginkgo biloba L. extracts and leaves can be routinely carried out by reversedphase HPLC (RP-HPLC) with evaporative light scattering detection (ELSD) (Dubber \& Kanfer, 2006; Kaur et al., 2009), refractive index (RI) (Wang \& Ju, 2000), diode array detector (DAD) (Tang et al., 2010) and mass spectrometry detection (Wang et al., 2017). As an orthogonal technique gas chromatography (GC) system equipped with a flame ionization detector (FID) or mass spectrometry (MS) detection (Czigle et al., 2019) is also used. At sample preparation steps; cold and hot extraction, pressurized solvent extraction and ultrasoundassisted extraction is typically applied to achieve the simultaneous analysis of terpene trilactones and flavonol aglycones (Kaur et al., 2009; Liu et al., 2015).

Apitherapy has been gaining great attention nowadays due to its superior health effects. Bee products like pollen, royal jelly, honey, and especially propolis are rich in flavonoids, phenolic acids, minerals, and diverse anti-inflammatory and immune booster substances. The antioxidant capacities, phenolic acids, and flavonoid contents of $P$. ginseng and Ginkgo biloba L. extracts mixed with bee products are expected to be increased significantly. This research aimed to determine and compare the bio-active phytochemical contents, antioxidant capacities, and phenolic profiles of $P$. ginseng and Ginkgo biloba L. extract products as well as their preparations in mixture with bee products applying developed methodologies. In addition to many studies that investigated the health benefits and pharmacological properties of bee products, our research addedly focused on the investigation of active substance diversities at commercially available supplements and tried to find answers to how apitherapy products may change the phenolic and antioxidant properties of $P$. ginseng and Ginkgo biloba L. extracts that have been used in traditional medicine for years. Moreover, a high-resolution phenolic profile determination method with a comprehensive compound library and using mass-based detection was targeted to be developed. Thanks to new methods in terms of ginsenoside, terpene lactone, and phenolic monitoring, we envisage that this research will contribute a lot to the literature for advanced phytotherapy research.

\section{MATERIAL and METHODS}

\subsection{Sample Collection}

Products containing $P$. ginseng extract $(\mathrm{n}=12)$ and samples containing Ginkgo biloba $\mathrm{L}$. extract $(\mathrm{n}=9)$ were purchased from the pharmacies and herbalists. Two of the samples that contain Ginkgo biloba L. extracts and seven of the samples containing $P$. ginseng extract were in form 
of mixtures with alternative bee products. Information of the samples regarding their serving sizes and their forms are given in Table 1.

Table 1. Forms and serving sizes of $P$. ginseng \& Ginkgo biloba L. samples.

\begin{tabular}{|c|c|c|c|c|c|}
\hline Product & Product Type/Form & Serving size & Product & $\begin{array}{c}\text { Product } \\
\text { Type/Form }\end{array}$ & Serving size \\
\hline $\mathrm{A}^{a}{ }^{a}$ & Paste & $7 \mathrm{~g}$ & B1 & Tablet & 1 tablet / $50 \mathrm{mg}$ \\
\hline $\mathrm{A} 2^{a}$ & Paste & $7 \mathrm{~g}$ & $\mathrm{~B} 2^{c}$ & Paste & $7 \mathrm{~g}$ \\
\hline $\mathrm{A} 3^{a}$ & Paste & $7 \mathrm{~g}$ & B3 & Liquid Extract & $7 \mathrm{~g}$ \\
\hline $\mathrm{A} 4^{a}$ & Tablet & 1 tablet / $780 \mathrm{mg}$ & B4 & Tablet & 1 tablet $/ 722 \mathrm{mg}$ \\
\hline $\mathrm{A} 5^{b}$ & Liquid Extract & $7 \mathrm{~g}$ & B5 & Capsule & 1 tablet / $654 \mathrm{mg}$ \\
\hline A6 & Tablet & 1 tablet / $722 \mathrm{mg}$ & $\mathrm{B} 6^{a}$ & Paste & $7 \mathrm{~g}$ \\
\hline A7 & Tablet & 1 tablet / $1770 \mathrm{mg}$ & B7 & Capsule & $\begin{array}{c}1 \text { capsule / } \\
\mathrm{mg}\end{array}$ \\
\hline $\mathrm{A} 8^{a}$ & Paste & $7 \mathrm{~g}$ & B8 & Tablet & 1 tablet $/ 870 \mathrm{mg}$ \\
\hline A9 & Capsule & 1 capsule / $670 \mathrm{mg}$ & B9 & Capsule & $\begin{array}{c}1 \text { capsule / } 450 \\
\text { mg }\end{array}$ \\
\hline $\begin{array}{l}\mathrm{A} 10 \\
\mathrm{~A} 11 \\
\mathrm{~A} 12^{a}\end{array}$ & $\begin{array}{c}\text { Capsule } \\
\text { Paste } \\
\text { Liquid Extract }\end{array}$ & $\begin{array}{c}1 \text { capsule } / 1560 \mathrm{mg} \\
7 \mathrm{~g} \\
25 \mathrm{~g}\end{array}$ & & & \\
\hline
\end{tabular}

A1-12: Panax ginseng C.A. Meyer products; B1-9: Ginkgo biloba L. products; ${ }^{\text {a }}$ Contains more than a single bee product; ${ }^{\mathbf{b}}$ Contains royal jelly $(\mathrm{RJ}) ;{ }^{\mathbf{c}}$ Contains honey

\subsection{Reagents and Chemicals}

The analytical standard mixture of Ginkgo biloba L. terpene lactones, containing bilobalide, ginkgolide A, ginkgolide $\mathrm{B}$, ginkgolide $\mathrm{C}$, ginkgolide $\mathrm{J}(0.1 \mathrm{mg} / \mathrm{ml}$ of each component in acetonitrile) was purchased from Cerilliant Corporation ${ }^{\circledR}$ (Round Rock, Texas, USA) and a standard mixture of ginsenosides that contain $\mathrm{Rg}_{2}, \mathrm{Rb}_{1}, \mathrm{Rb}_{2}, \mathrm{Rc}, \mathrm{Re}, \mathrm{Rd}, \mathrm{Rg}_{1}$ and $\mathrm{Rf}(0.1 \mathrm{mg} / \mathrm{ml}$ of each component in methanol) were also obtained from Cerilliant Corporation ${ }^{\circledR}$ (Round Rock, Texas, USA). Individual standards of ginsenoside $\mathrm{Ra}_{1}, \mathrm{Ra}_{3}$, and $\mathrm{Rb}_{3}$ were supplied from Fleton Natural Products Co., Ltd $^{\circledR}$ (Chengdu, China). A second working stock standard mixture for additional ginsenosides ( $\mathrm{Ra} 1, \mathrm{Ra} 3$, and $\mathrm{Rb} 3$ ) was prepared by dissolving them in methanol at $0.1 \mathrm{mg} / \mathrm{ml}$ concentrations. The analytical standards of each phenolic substances (Cinnamyl aldehyde, 3,4-dimethoxy benzaldehyde, phloroglucinol, 4-hydroxybenzoic acid, transcinnamic acid, protocatechuic acid, p-coumaric acid, 2-OH-coumaric acid, m-coumaric acid, phenyllactic acid, vanillic acid, homogentisic acid, gallic acid, shikimic acid, caffeic acid, quinic acid, ferulic acid, syringic acid, 3,4-dimethoxy cinnamic acid, methylsyringate, resveratrol, chrysin, pinocembrin, apigenin, genistein, galangin, naringenin, pinobanksin, caffeic acid phenethyl ester (CAPE), ellagic acid, kaempferol, luteolin, epicatechin, catechin, quercetin, hesperetin, taxifolin, epigallocatechin, isorhamnetin, chlorogenic acid, rutin, myricetin, and rosmarinic acid) were purchased from Sigma-Aldrich ${ }^{\circledR}$ (Munich, Germany). Stock standards of each phenolic substances were prepared by dissolving them in ethanol individually at $10 \mathrm{mg} / \mathrm{ml}$ concentration. For calibration purposes, a phenolic stock standard mixture at $0.01 \mathrm{mg} / \mathrm{ml}$ was prepared by adding $200 \mu \mathrm{l}$ from each phenolic stock standard in a falcon tube and diluting it to an appropriate volume. Afterward, the phenolic stock standard mixture was diluted for the constitution of the 6 linear calibration points $(0.05 \mu \mathrm{g} / \mathrm{ml}, 0.1 \mu \mathrm{g} / \mathrm{ml}, 0.25 \mu \mathrm{g} / \mathrm{ml}, 0.5 \mu \mathrm{g} / \mathrm{ml}, 1.0$ $\mu \mathrm{g} / \mathrm{ml}$, and $2.0 \mu \mathrm{g} / \mathrm{ml}$ ) using deionized water, thereby external standard calibration plots with the range from $0.05 \mu \mathrm{g} / \mathrm{ml}$ to $2.0 \mu \mathrm{g} / \mathrm{ml}$ concentrations were obtained for each phenolic compound. DPPH (2,2-diphenyl-1-picrylhydrazyl) was also purchased from Sigma-Aldrich ${ }^{\circledR}$. $19.7 \mathrm{mg}$ DPPH was dissolved in $25 \mathrm{ml}$ ethanol and used as reagent at free radical scavenging activity assays. Acetonitrile (ACN), acetic acid, ethanol (EtOH, LC Grade) was obtained from 
Merck $^{\circledR}$ (Darmstadt, Germany). Methanol (MeOH) was obtained from JT. Baker ${ }^{\circledR}$ (Deventer, Holland). Ultrapure water was taken from a Milli-Q ${ }^{\circledR}$ Plus system in our laboratory (Millipore ${ }^{\circledR}$, Billerica, MA, USA).

\subsection{Terpene Lactone Analysis}

$100 \mathrm{mg}$ of Ginkgo biloba L. product was weighed in a $50 \mathrm{ml}$ falcon tube and $20 \mathrm{ml}$ of $90 \%$ $\mathrm{MeOH}$ solution was added. The sample was extracted using an ultrasonic bath (Sonorex ${ }^{\circledR} \mathrm{RK}$ $154 \mathrm{BH}$, Bandelin Electronic ${ }^{\circledR} \mathrm{GmbH} \& \mathrm{Co} . \mathrm{KG}$, Berlin, Germany) at $50^{\circ} \mathrm{C}$ for $15 \mathrm{~min}$. It was then centrifugated at $3000 \mathrm{rpm}$ for $10 \mathrm{~min}$. (Hettich ${ }^{\circledR}$, Rotina 35R Type, Tuttlingen, Germany) and the supernatant was transferred to a new falcon tube. This extraction step was repeated twice more. Transferred supernatants were combined $(\sim 50 \mathrm{ml})$ vortexed-mixed and completed to a volume of $50 \mathrm{ml}$ with deionized water. Prepared samples were transferred to glass vial by diluting 1:10 (w/v) with deionized water. Calibration standards at $0.5,1.0,2.0$, and $4.0 \mu \mathrm{g} / \mathrm{ml}$ were also prepared by diluting the standard mixture solution of terpene lactones using deionized water. All standards and samples were kept at $4^{\circ} \mathrm{C}$ until analysis. The chromatographic analysis was performed on Waters ${ }^{\circledR}$ ACQUITY UPLC (Waters ${ }^{\circledR}$, Milford, MA, USA) system equipped with a binary solvent delivery system and autosampler. The chromatographic separation was achieved on Waters ${ }^{\circledR}$ ACQUITY UPLC HSS (High Strength Silica) T3 column $(2.1 \mathrm{~mm}$ x 75 $\mathrm{mm}, 1.8 \mu \mathrm{m}$ ). The mobile phase consists of solution A (water containing $0.005 \%$ acetic acid) and solution $\mathrm{B}$ (methanol containing $0.005 \%$ acetic acid). The gradient elution was optimized as follows: $10 \% \mathrm{~B}(0-0.5 \mathrm{~min}$.), $10-70 \% \mathrm{~B}(0.5-7 \mathrm{~min}$.), $70-10 \% \mathrm{~B}(7-7.01 \mathrm{~min}$.), $10 \% \mathrm{~B}$ (7.01-8 $\mathrm{min}$.). The flow rate was $0.35 \mathrm{ml} / \mathrm{min}$. The column and autosampler temperatures were maintained at $40^{\circ} \mathrm{C}$ and $4{ }^{\circ} \mathrm{C}$, respectively. The injection volume was $10 \mu \mathrm{L}$. The detection was done via Waters ${ }^{\circledR}$ Xevo TQ tandem quadrupole mass spectrometer (Micromass ${ }^{\circledR}$ MS Technologies, Manchester, UK) with electrospray ionization (ESI) interface in multiple reaction monitoring $(\mathrm{MRM})$ and positive ionization mode. The needle capillary voltage was set at $3 \mathrm{kV}$. The flow rate and temperature of desolvation gas were $850 \mathrm{~L} / \mathrm{h}$ and $450{ }^{\circ} \mathrm{C}$, respectively. The flow rate of cone gas was $50 \mathrm{~L} / \mathrm{h}$ and source temperature operated at $150^{\circ} \mathrm{C}$. These conditions were optimized by analyzing the reference compounds and comparing the signal-to-noise ratio $(\mathrm{S} / \mathrm{N})$ for each analyte. All ESI and mass spectrometer conditions were optimized individually for each target compound and listed in Table 2. Dwell times were automatically set by the software. Data acquisitions and quantifications were performed using Waters ${ }^{\circledR}$ Mass-Lynx software with the Target Lynx program.

Table 2. MRM transitions and mass spectrometry parameters for terpene lactone analysis.

\begin{tabular}{lccccc}
\hline $\begin{array}{c}\text { Analyte / Ionization } \\
\text { Mode (+/-) }\end{array}$ & $\begin{array}{c}\text { Precursor Ion } \\
(\mathrm{m} / \mathrm{z})\end{array}$ & $\begin{array}{c}\text { Product Ion } \\
(\mathrm{m} / \mathrm{z})\end{array}$ & $\begin{array}{c}\text { Dwell Time } \\
(\mathrm{sec} .)\end{array}$ & $\begin{array}{c}\text { Cone Voltage } \\
(\mathrm{V})\end{array}$ & $\begin{array}{c}\text { Collision } \\
\text { Energy }(\mathrm{eV})\end{array}$ \\
\hline Bilobalide / + & 325.1 & 163.0 & 0.005 & 30 & 20 \\
Ginkgolide A / + & 407.1 & 350.9 & 0.005 & 50 & 15 \\
Ginkgolide B / + & 423.1 & 367.1 & 0.005 & 22 & 16 \\
Ginkgolide C / + & 438.5 & 382.9 & 0.005 & 30 & 16 \\
Ginkgolide J / & 469.0 & 423.0 & 0.005 & 30 & 15 \\
\hline
\end{tabular}

\subsection{Ginsenoside Analysis}

$500 \mathrm{mg}$ of $P$. ginseng product was weighed and $15 \mathrm{ml}$ of $90 \% \mathrm{MeOH}$ was added. Ultrasoundassisted extraction was performed at $50^{\circ} \mathrm{C}$ for $15 \mathrm{~min}$. The sample was centrifugated at 3000 rpm for $10 \mathrm{~min}$. After the transfer of supernatant to a new falcon tube, the extraction process 
was repeated two more times and all supernatants were combined ( $\sim 45 \mathrm{ml})$ after each extraction step. Extracts were vortexed-mixed and completed to volume of $50 \mathrm{ml}$. Final prepared samples were transferred to glass vial by diluting $1: 10(\mathrm{w} / \mathrm{v})$ with ultra-pure water. Calibration standards at $0.5,1.0,2.0$, and $4.0 \mu \mathrm{g} / \mathrm{ml}$ concentrations were prepared by appropriate dilution of the standard mix. solutions of ginsenosides. Waters ${ }^{\circledR}$ ACQUITY UPLC system equipped with a binary solvent delivery system and autosampler was used to perform the separation. A chromatographic resolution was crucial and this was achieved using a Waters ${ }^{\circledR}$ Acquity BEH (Bridge Ethylene Hybrid) C18 column $(2.1 \mathrm{~mm}$ x $50 \mathrm{~mm}, 1.7 \mu \mathrm{m})$. The liquid chromatography phases consisted of water as mobile phase $A$ and acetonitrile $(\mathrm{ACN})$ as mobile phase $\mathrm{B}$ without any modifiers. The gradient elution was optimized as follows: $15 \% \mathrm{~B}(0-1 \mathrm{~min}),. 15-35 \% \mathrm{~B}$ (1-9 min.), 35-75\% B (9-12 min.), 75-15\% B (12-12.01 min.), 15\% B (12.01-14 min.). The flow rate was $0.4 \mathrm{ml} / \mathrm{min}$. The column oven set to $40^{\circ} \mathrm{C}$ and autosampler temperature was maintained at $4{ }^{\circ} \mathrm{C}$. The injection volume was $10 \mu \mathrm{l}$. The high accuracy mass spectrometric data were recorded on Waters ${ }^{\circledR}$ Xevo TQ tandem quadrupole mass spectrometer with ESI source in MRM mode. The optimized MS parameters were as follows: the capillary voltage: $3 \mathrm{kV}$, flow rate, and temperature of desolvation gas: $800 \mathrm{~L} / \mathrm{h}$ and $450{ }^{\circ} \mathrm{C}$ respectively, flow rate of cone gas: $50 \mathrm{~L} / \mathrm{h}$, and source temperature: $150^{\circ} \mathrm{C}$. Ionization states and MS parameters were optimized individually for each target compound and they were listed in Table 3. Dwell times were automatically set by the Mass-Lynx software. Data acquisition was controlled by the Target Lynx software from Waters ${ }^{\circledR}$.

Table 3. MRM transitions and mass spectrometry parameters for ginsenoside analysis.

\begin{tabular}{|c|c|c|c|c|c|}
\hline $\begin{array}{c}\text { Analyte / } \\
\text { Ionization Mode } \\
(+/-)\end{array}$ & $\begin{array}{l}\text { Precursor Ion } \\
(\mathrm{m} / \mathrm{z})\end{array}$ & $\begin{array}{l}\text { Product Ion } \\
(\mathrm{m} / \mathrm{z})\end{array}$ & $\begin{array}{l}\text { Dwell Time } \\
\text { (sec.) }\end{array}$ & $\begin{array}{l}\text { Cone Voltage } \\
\text { (V) }\end{array}$ & $\begin{array}{c}\text { Collision Energy } \\
(\mathrm{eV})\end{array}$ \\
\hline $\mathrm{Ra}_{1} /-$ & 1209.0 & 945.0 & 0.005 & 80 & 40 \\
\hline $\mathrm{Ra}_{1} /-$ & 1209.0 & 1077.0 & 0.005 & 80 & 40 \\
\hline $\mathrm{Rg}_{2} /-$ & 783.0 & 391.0 & 0.005 & 85 & 40 \\
\hline $\mathrm{Rg}_{2} /-$ & 783.0 & 475.0 & 0.005 & 85 & 40 \\
\hline $\mathrm{Rg}_{2} /-$ & 783.0 & 637.0 & 0.005 & 85 & 40 \\
\hline $\mathrm{Ra}_{3} /+$ & 1263.0 & 437.0 & 0.005 & 80 & 50 \\
\hline $\mathrm{Ra}_{3} /+$ & 1263.0 & 497.0 & 0.005 & 80 & 50 \\
\hline $\mathrm{Ra}_{3} /+$ & 1263.0 & 789.0 & 0.005 & 80 & 50 \\
\hline $\mathrm{Rb}_{1} /+$ & 1131.0 & 305.0 & 0.005 & 85 & 55 \\
\hline $\mathrm{Rb}_{1} /+$ & 1131.0 & 365.0 & 0.005 & 85 & 55 \\
\hline $\mathrm{Rb}_{1} /+$ & 1131.0 & 789.4 & 0.005 & 85 & 55 \\
\hline $\mathrm{Rb}_{1} /+$ & 1131.0 & 772.1 & 0.005 & 85 & 55 \\
\hline $\mathrm{Rb}_{2}+\mathrm{Rb}_{3} /+$ & 1101.7 & 789.4 & 0.005 & 90 & 55 \\
\hline $\mathrm{Rb}_{2}+\mathrm{Rb}_{3} /+$ & 1101.8 & 335.0 & 0.005 & 85 & 55 \\
\hline $\mathrm{Rc} /+$ & 1101.0 & 335.0 & 0.005 & 80 & 50 \\
\hline $\mathrm{Rc} /+$ & 1101.7 & 789.4 & 0.005 & 85 & 50 \\
\hline $\operatorname{Re} /+$ & 969.6 & 203.2 & 0.005 & 85 & 55 \\
\hline $\operatorname{Re} /+$ & 969.6 & 349.4 & 0.005 & 85 & 55 \\
\hline $\mathrm{Rd} /+$ & 969.0 & 365.0 & 0.005 & 85 & 50 \\
\hline $\mathrm{Rd} /+$ & 969.6 & 789.4 & 0.005 & 85 & 50 \\
\hline $\operatorname{Rg}_{1} /+$ & 823.5 & 203.2 & 0.005 & 85 & 50 \\
\hline $\operatorname{Rg}_{1} /+$ & 823.5 & 643.3 & 0.005 & 85 & 50 \\
\hline $\mathrm{Rf} /+$ & 823.0 & 365.0 & 0.005 & 80 & 50 \\
\hline $\mathrm{Rf} /+$ & 823.0 & 424.0 & 0.005 & 80 & 50 \\
\hline
\end{tabular}




\subsection{Phenolic Profiling}

$1.0 \mathrm{~g}$ of all products was weighed and $30 \mathrm{ml} \mathrm{70 \%} \mathrm{ethanol} \mathrm{(EtOH)} \mathrm{solution} \mathrm{was} \mathrm{added} \mathrm{for}$ extraction. Samples were extracted by shaking overnight using a rotary orbital shaker (Thermo Fisher ScientificTM, Inc.MaxQ 4000 Benchtop Orbital Shaker, Waltham, MA, USA). It was centrifugated at $3000 \mathrm{rpm}$ for $5 \mathrm{~min}$. and the supernatant was separated. Extraction was repeated and the total volume of supernatants was completed to $100 \mathrm{ml}$ with EtOH. Samples were diluted to appropriate volume depending on the phenolic concentration of the sample to be able to quantify between the calibration range with deionized water and filtered to glass vials using a $0.45 \mu \mathrm{m}$ PVDF filter (Interlab ${ }^{\circledR}$, Istanbul, Turkey) prior to chromatographic analysis. Calibration standards at $0.05,0.1,0.25,0.5,1.0$ and $2.0 \mu \mathrm{g} / \mathrm{ml}$ were prepared from stock standard mixture by applying serial dilutions. The chromatographic system was Waters ${ }^{\circledR}$ ACQUITY UPLC, which consisted of a binary solvent delivery system and autosampler. The separation was performed on Waters ${ }^{\circledR}$ CORTECS T3 column $(1,6 \mu \mathrm{m} \mathrm{2,1}$ x $150 \mathrm{~mm})$ using a gradient elution of (A) water containing 0.01\% acetic acid and (B) 80:20 ACN:MeOH containing 0.01 acetic acid at a flow rate of $0.25 \mathrm{ml} / \mathrm{min}$. Gradient elution was applied as follows: $2 \%$ B (0-1.30 min.), 2-55\% B (1.30-35 min.), 55-95\% B (35-37 min.), 95-2\% B (37-37.01 min.), 2\% B (37.01-40 min.). The column and autosampler tray temperatures were $30^{\circ} \mathrm{C}$ and $10^{\circ} \mathrm{C}$, respectively. The effluent from the LC outlet was directed into the ionization electrospray source of Waters ${ }^{\circledR}$ Xevo TQ tandem quadrupole mass spectrometer after $1.0 \mathrm{~min}$. delay using the embedded valve on the device. The ion source and desolvation temperature were held at $150{ }^{\circ} \mathrm{C}$ and $450{ }^{\circ} \mathrm{C}$, respectively. The flow rate of desolvation and cone gas were optimized at $850 \mathrm{~L} / \mathrm{h}$ and $50 \mathrm{~L} / \mathrm{h}$ respectively. The needle capillary voltage was determined at $2 \mathrm{kV}$ for ideal responses. MRM mode was employed for analysis. Peak areas for all components were automatically integrated using Mass-Lynx software with the Target Lynx program $\left(\right.$ Waters ${ }^{\mathbb{B}}$ ). All ESI and MS parameters were optimized individually for each target compound and listed in Table 4.

Table 4. MRM transitions and mass spectrometry parameters for phenolic compound analysis.

\begin{tabular}{lllllc}
\hline Analyte & $\begin{array}{l}\text { Precursor } \\
\text { Ion } \\
(\mathrm{m} / \mathrm{z})\end{array}$ & Product Ions $(\mathrm{m} / \mathrm{z})$ & $\begin{array}{l}\text { Cone } \\
\text { Voltage } \\
(\mathrm{V})\end{array}$ & $\begin{array}{l}\text { Collision } \\
\text { Energy }(\mathrm{eV})\end{array}$ & $\begin{array}{c}\text { Ionization } \\
\text { Mode } \\
(+/-)\end{array}$ \\
\hline Cinnamyl Aldehyde & 133.2 & $55.0 / 77.2 / 105.2$ & 20 & $15 / 20 / 15$ & + \\
3,4 Dimethoxy & 167.0 & $124.0 / 139.2$ & 20 & $15 / 15$ & + \\
Benzaldehyde & & & & & + \\
Phloroglucinol & 124.9 & $56.9 / 82.8$ & 25 & $15 / 15$ & - \\
4-Hydroxybenzoic Acid & 137.0 & 92.9 & 20 & 15 & - \\
Transcinnamic Acid & 147.0 & $77.0 / 102.8$ & 25 & $20 / 20$ & - \\
Protocatechunic Acid & 152.9 & 108.8 & 25 & 15 & - \\
p-Coumaric Acid & 163.0 & $93.0 / 119.0 / 147.0$ & 25 & $20 / 20 / 20$ & - \\
2-OH Coumaric Acid & 163.0 & $93.0 / 119.0 / 147.0$ & 25 & $20 / 20 / 20$ & - \\
m-Coumaric Acid & 163.0 & $93.0 / 119.0 / 147.0$ & 25 & $20 / 20 / 20$ & - \\
Phenyllactic Acid & 165.1 & $102.8 / 118.9 / 146.9$ & 25 & $15 / 15 / 10$ & - \\
Vanilic Acid & 166.9 & $90.8 / 108.1 / 123.2 / 152.2$ & 25 & $20 / 20 / 10 / 25$ & - \\
Homogentisic Acid & 167.0 & $122.9 / 123.1$ & 20 & $20 / 20$ & - \\
Gallic Acid & 169.0 & 124.9 & 25 & 20 & - \\
Shikimic Acid & 173.0 & $73.0 / 93.0 / 111.0$ & 25 & $20 / 20 / 20$ & - \\
Caffeic Acid & 179.0 & 135.0 & 25 & 20 & - \\
Quinic Acid & 191.1 & $59.0 / 84.8 / 92.8 / 126.8$ & 35 & $20 / 20 / 20 / 20$ & - \\
Ferrulic Acid & 193.0 & $134.0 / 149.0 / 178.0$ & 25 & $20 / 20 / 20$ & - \\
\hline
\end{tabular}


Table 4. Continues.

\begin{tabular}{|c|c|c|c|c|c|}
\hline Syringic Acid & 197.0 & $123.0 / 167.0 / 182.0$ & 25 & $20 / 20 / 20$ & - \\
\hline $\begin{array}{l}\text { 3,4 Dimethoxycinnamic } \\
\text { Acid }\end{array}$ & 206.7 & 102.7 & 25 & 20 & - \\
\hline Methylsyringate & 211.2 & $181.0 / 196.0$ & 25 & $20 / 20$ & - \\
\hline Quercetin & 301.0 & $150.8 / 178.9$ & 35 & $20 / 20$ & - \\
\hline Ellagic Acid & 301.0 & $185.2 / 229.0 / 257.0 / 284.2$ & 30 & $30 / 25 / 25 / 30$ & - \\
\hline Hesperetin & 301.3 & $135.8 / 150.8 / 164.1 / 241.7$ & 25 & $20 / 20 / 20 / 20$ & - \\
\hline Taxifolin & 303.0 & 125.0 & 25 & 20 & - \\
\hline Epigallocatechin & 305.2 & $124.8 / 164.8 / 166.9 / 219.0$ & 25 & $20 / 20 / 20 / 20$ & - \\
\hline Isorhamnetin & 315.0 & 300.0 & 25 & 20 & - \\
\hline Myricetin & 317.0 & $137.2 / 151.2 / 179.2$ & 35 & $25 / 25 / 20$ & - \\
\hline Chlorgenic Acid & 353.3 & $179.0 / 191.0$ & 25 & $20 / 20$ & - \\
\hline Rosmarinic Acid & 359.0 & $161.0 / 197.0$ & 25 & $20 / 20$ & - \\
\hline Rutin & 609.1 & $300.0 / 301.0$ & 25 & $20 / 20$ & - \\
\hline Resveratrol & 227.0 & $143.0 / 185.0$ & 30 & $20 / 20$ & - \\
\hline Chrysin & 253.0 & $151 / 209 / 225$ & 25 & $20 / 20 / 20$ & - \\
\hline Pinocembrin & 255.0 & $151.0 / 171.0 / 213.0$ & 25 & $20 / 20 / 20$ & - \\
\hline Apigenin & 269.0 & $117.3 / 149.0 / 151.0$ & 40 & $30 / 25 / 25$ & - \\
\hline Genistein & 269.0 & $133.2 / 159.2 / 224.2 / 240.0$ & 40 & $30 / 20 / 25 / 20$ & - \\
\hline Galangin & 269.0 & $197.0 / 213.0 / 227.0$ & 25 & $20 / 20 / 20$ & - \\
\hline Naringenin & 271.0 & $145.0 / 151.0$ & 25 & $20 / 20$ & - \\
\hline Pinobanksin & 271.2 & $153.0 / 225.0 / 253.0$ & 25 & $20 / 20 / 20$ & - \\
\hline CAPE & 283.0 & $135.0 / 161.0 / 179.0$ & 25 & $20 / 20 / 20$ & - \\
\hline Kaempferol & 285.0 & $93.0 / 151.0 / 257.0$ & 25 & $20 / 20 / 20$ & - \\
\hline Luteolin & 285.0 & $133.0 / 241.0 / 267.0$ & 25 & $20 / 20 / 20$ & - \\
\hline Epicatechin & 289.1 & $108.8 / 203.0 / 245.0$ & 25 & $20 / 20 / 20$ & - \\
\hline Catechin & 289.1 & $108.8 / 203.0 / 245.0$ & 25 & $20 / 20 / 20$ & - \\
\hline
\end{tabular}

\subsection{Free Radical Scavenging Activity (Antioxidant Capacity Assay)}

The percentage of antioxidant activity (AA\%) of each sample was assessed by DPPH free radical scavenging assay. Hydroalcoholic extracts prepared for phenolic profiling analysis were used as representative samples and reacted with the reagent of DPPH radical. For this, $2.3 \mathrm{ml}$ of sample extract was mixed with $0.3 \mathrm{ml}$ of DPPH solution $(0.1 \mathrm{mmol} / \mathrm{L})$ and it was incubated in the dark for $15 \mathrm{~min}$. The changes in color (from deep violet to light yellow) were observed and the absorbance of sample solutions was read at $517 \mathrm{~nm}$ against ethanol/DPPH solution as a reagent blank using a UV-VIS spectrophotometer (Thermo Fisher Scientific ${ }^{\circledR}$ Inc., Electron Evolution 300 UV-VIS Spectrophotometer, Waltham, MA, USA).

\section{RESULTS and DISCUSSION}

\subsection{Ginsenosides}

12 commercial samples were gathered and analyzed using the developed Ultra Performance Liquid Chromatography Multiple Reaction Monitoring Mass Spectrometry (UPLC/MRM-MS) method for ginsenoside contents. Thanks to our high-resolution chromatographic separation method, as shown in Figure 1, ginsenoside molecules were resolved and analyzed in high specificity and in short analysis time. Only the ginsenoside $\mathrm{Rb} 2$ and ginsenoside $\mathrm{Rb} 3$ components were co-eluted and their concentrations are reported as total amount. 
The majority of these samples are in mixture with different bee products; A1: pollen and honey, A2: pollen, honey, propolis, and royal jelly, A3: royal jelly and propolis, A4: pollen, royal jelly, and propolis, A5: royal jelly A8: propolis and honey A12: propolis, royal jelly, and honey. All quantified results were converted to their corresponding percentage for each ginsenoside component in the $P$. ginseng sample. The analysis results are given in Table 5.

Table 5. Analysis results of the ginsenoside contents.

\begin{tabular}{|c|c|c|c|c|c|c|}
\hline Sample & Label claim & $\begin{array}{l}\text { Serving } \\
\text { size }\end{array}$ & $\begin{array}{l}\text { Ginsenoside } \\
\text { percentage of } \\
\text { the product } \\
(\mathrm{w} / \mathrm{w} \%) \\
(\mathrm{mg} / 100 \mathrm{mg})\end{array}$ & $\begin{array}{c}\text { Total } \\
\text { Ginsenoside } \\
\text { content at per } \\
\text { serving }(\mathrm{mg})\end{array}$ & $\begin{array}{l}\text { Recommended } \\
\text { daily serving } \\
\text { amount }\end{array}$ & $\begin{array}{c}\text { Quantified } \\
\text { daily intake } \\
\text { amounts of } \\
\text { Total } \\
\text { Ginsenosides } \\
\text { (mg) } \\
\end{array}$ \\
\hline $\mathrm{A} 1^{*}$ & - & $7 \mathrm{~g}$ & 0.16 & 11.19 & $7 \mathrm{~g} \times 3$ & 33.57 \\
\hline $\mathrm{A} 2^{*}$ & - & $7 \mathrm{~g}$ & 0.00 & 0.00 & $7 \mathrm{~g} \times 3$ & 0.00 \\
\hline $\mathrm{A} 3^{*}$ & $\begin{array}{l}80 \mathrm{mg} \text { of Ginseng } \\
\text { Powder Extract }+ \\
20 \mathrm{mg} \text { of } \\
\text { Ginsenosides at } \\
\text { per serving }\end{array}$ & $7 \mathrm{~g}$ & 1.25 & 87.47 & $7 \mathrm{~g} \mathrm{x} 1$ & 87.47 \\
\hline $\mathrm{A} 4^{*}$ & $\begin{array}{l}235 \mathrm{mg} \text { of Panax } \\
\text { Ginseng Extract at } \\
\text { per tablet }\end{array}$ & $\begin{array}{l}1 \text { tablet } \\
(780 \mathrm{mg})\end{array}$ & 2.12 & 16.51 & 2 tablet & 33.02 \\
\hline $\mathrm{A} 5^{*}$ & - & $7 \mathrm{~g}$ & 0.14 & 9.71 & $7 \mathrm{~g} \times 3$ & 29.13 \\
\hline A6 & $\begin{array}{l}26,96 \mathrm{mg} \text { of } \\
\text { Ginsenosides }+49 \\
\text { mg of Ginseng } \\
\text { Granul Extract }+ \\
490,3 \mathrm{mg} \text { of } \\
\text { Ginseng Powder } \\
\text { Extract }\end{array}$ & $\begin{array}{l}1 \text { tablet } \\
\text { (722 mg) }\end{array}$ & 11.18 & 80.70 & 1 tablet & 80.70 \\
\hline A7 & $\begin{array}{l}50 \mathrm{mg} \text { of Korean } \\
\text { Ginseng Extract at } \\
\text { per serving }\end{array}$ & $\begin{array}{c}1 \text { tablet } \\
(1770 \\
\mathrm{mg})\end{array}$ & 0.96 & 16.91 & 1 tablet & 16.91 \\
\hline $\mathrm{A} 8^{*}$ & $\begin{array}{l}12 \mathrm{mg} \text { of } \\
\text { Ginsenosides per } \\
\text { serving }\end{array}$ & $7 \mathrm{~g}$ & 0.17 & 12.05 & $7 \mathrm{~g} \mathrm{x} 3$ & 36.15 \\
\hline A9 & $\begin{array}{l}500 \mathrm{mg} \text { of Korean } \\
\text { Ginseng Powder } \\
\text { Extract: } 28,8 \mathrm{mg} \text { of } \\
\text { Total Ginsenosides }\end{array}$ & $\begin{array}{l}1 \text { capsule } \\
(670 \mathrm{mg})\end{array}$ & 5.19 & 29.04 & 1 tablet & 29.04 \\
\hline A10 & $\begin{array}{l}40 \text { mg of Panax } \\
\text { Ginseng Root } \\
\text { extract }\end{array}$ & $\begin{array}{c}1 \text { capsule } \\
(1560 \\
\mathrm{mg}) \\
\end{array}$ & 1.90 & 13.30 & 1 tablet & 13.30 \\
\hline A11 & - & $7 \mathrm{~g}$ & 0.33 & 23.28 & $7 \mathrm{~g} \mathrm{x} 1$ & 23.28 \\
\hline $\mathrm{A} 12^{*}$ & $\begin{array}{l}\% 5 \text { Red Ginseng } \\
\text { extract at per } \\
\text { serving }\end{array}$ & $25 \mathrm{~g}$ & 0,60 & 150.22 & $25 \mathrm{ml} \mathrm{x} 1$ & 150.22 \\
\hline
\end{tabular}

* Samples are in form of a mixture with apitherapy products. 
Figure 1. The chromatograms of ginsenosides standard mixture at $0.5 \mu \mathrm{g} / \mathrm{ml}$ concentration.

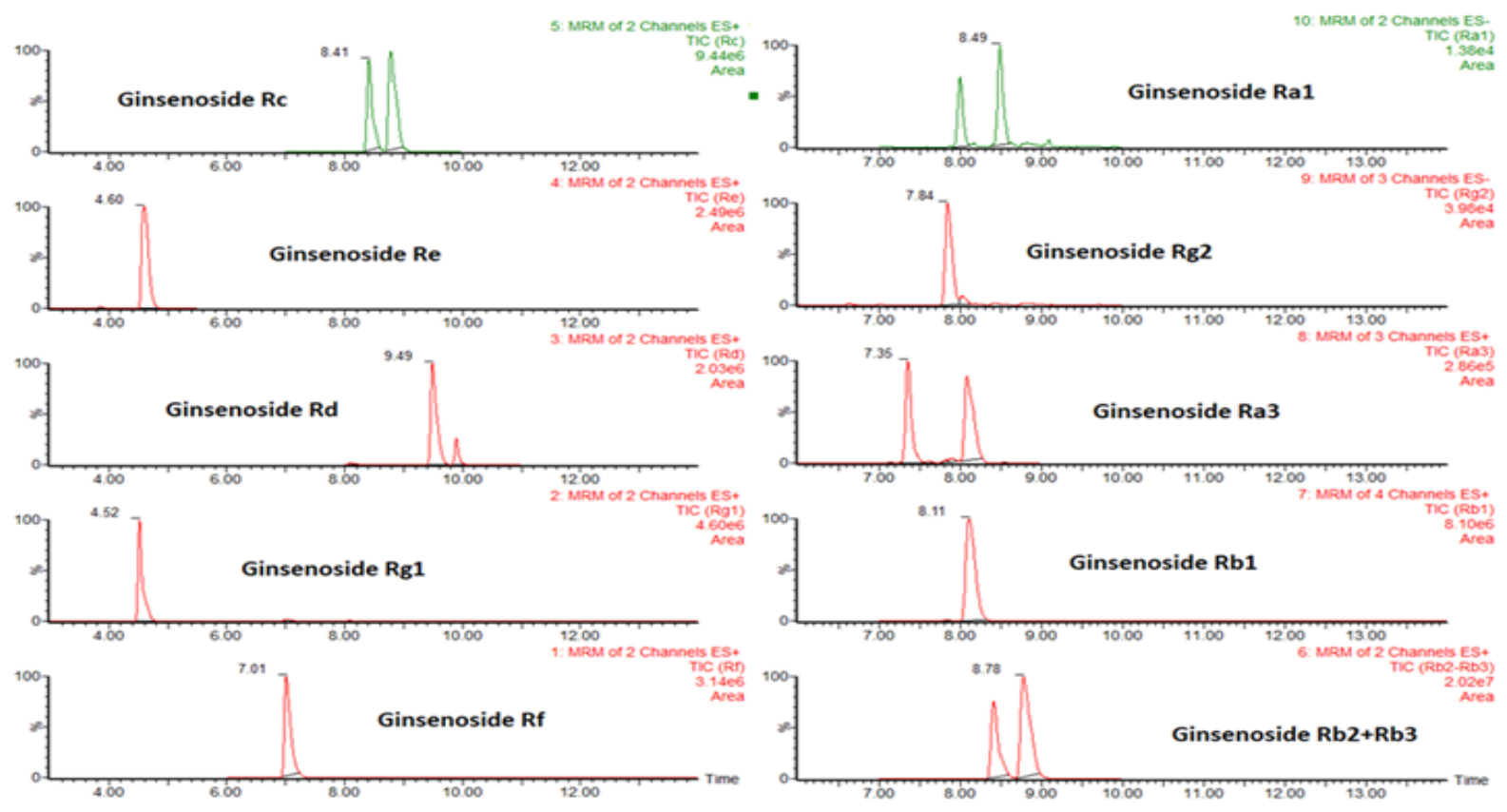

Total ginsenoside percentage values in the products were ranged between $0.14 \%$ and $11.80 \%$. Label fraud has been detected at A2 coded sample, since the ginsenoside content could not be quantified as a result of the analysis. Approximate percentages of total ginsenoside contents were similar at investigated samples except for A6 and A12 coded samples which have considerably higher quantities for daily intake. In addition to the total ginsenoside content, the compound diversity of the product and amount of each separate ginsenoside molecule is also a very important point of evaluation regarding the raw material quality. Considering that the therapeutic effects of each ginsenoside compound may vary, the diversity of ginsenoside types in $P$. ginseng extracts is a matter to be considered. In the samples coded A9 and A10, the percentage distribution of the compounds can be seen as homogeneous, while Ginsenoside Rd was detected as the predominant substance over the other components in 6 of the analyzed samples (A1, A3, A4, A7, A8, A10). Ginsenoside Re, Ginsenoside Rb2, and Rb3 were other high abundance ginsenoside species at A1, A3 and A4 coded samples alongside a high level of Ginsenoside Rd. While the highest content of ginsenoside in A5 coded sample was determined as $\mathrm{Rg} 2$, ginsenoside Ra1 with $\mathrm{Rg} 2$ in sample coded A6 stands out as the most dominant species. In the sample coded $\mathrm{A} 8$, in addition to the ginsenoside $\mathrm{Rd}, \mathrm{Rb} 2, \mathrm{Rb} 3$ components, unlike other samples, high levels of ginsenoside Rb1 and ginsenoside Rc were detected. In the A11 coded sample, ginsenoside $\operatorname{Rg} 1$ and ginsenoside Re components were in high amount, while in the A12 coded sample, ginsenoside Rc and ginsenoside Rb1 molecules were detected at high levels. As can be seen from the results, the ginsenoside types in the samples differ greatly due to factors such as extraction technique and ginseng types and/or origins. Results regarding the ginsenoside compound diversity are given in Table 6 . Whether the products include apitherapy additives or not, they were observed that $P$. ginseng contents were appropriate for recommended daily consumption, and except for one sample, all products mostly meet the values stated on the labels. Daily intake amounts of the products were ranged between $13.3 \mathrm{mg} /$ day and 150.2 $\mathrm{mg} /$ day. The recommended daily intake amounts were at the highest levels in two samples containing $P$. ginseng which are mixed with bee products (A3: $87.47 \mathrm{mg}$ and A12: $150.20 \mathrm{mg}$ ). 
Table 6. Analysis results of ginsenoside diversities by percentage.

Ginsenoside compounds by percentage (w/w \%) in total ginsenoside composition

\begin{tabular}{ccccccccccc}
\hline Samples & $\mathrm{Rf}$ & $\mathrm{Rg} 1$ & $\mathrm{Rd}$ & $\mathrm{Re}$ & $\mathrm{Rc}$ & $\mathrm{Rb} 2+\mathrm{Rb} 3$ & $\mathrm{Rb} 1$ & $\mathrm{Ra} 3$ & $\mathrm{Rg} 2$ & $\mathrm{Ra} 1$ \\
\hline $\mathrm{A} 1$ & 0.3 & 4.8 & 38.6 & 21.2 & 6.8 & 19.6 & 7.1 & 0.3 & 0.0 & 1.3 \\
$\mathrm{~A} 2$ & 0.0 & 0.0 & 0.0 & 0.0 & 0.0 & 0.0 & 0.0 & 0.0 & 0.0 & 0.0 \\
$\mathrm{~A} 3$ & 2.2 & 9.0 & 37.4 & 17.9 & 2.5 & 12.0 & 6.3 & 3.3 & 9.2 & 0.2 \\
$\mathrm{~A} 4$ & 1.0 & 9.8 & 30.6 & 16.8 & 7.5 & 17.3 & 7.4 & 0.6 & 6.9 & 2.2 \\
$\mathrm{~A} 5$ & 3.0 & 0.0 & 19.3 & 0.0 & 0.0 & 0.0 & 0.0 & 0.0 & 77.8 & 0.0 \\
$\mathrm{~A} 6$ & 0.2 & 3.4 & 15.7 & 6.9 & 4.0 & 11.2 & 2.5 & 0.1 & 26.0 & 29.9 \\
$\mathrm{~A} 7$ & 0.8 & 8.3 & 41.4 & 17.5 & 9.1 & 18.0 & 4.3 & 0.0 & 0.0 & 0.5 \\
$\mathrm{~A} 8$ & 4.5 & 2.7 & 21.8 & 9.9 & 18.5 & 16.4 & 20.6 & 5.7 & 0.0 & 0.0 \\
$\mathrm{~A} 9$ & 10.8 & 10.5 & 10.6 & 10.9 & 7.6 & 9.2 & 9.6 & 13.4 & 8.7 & 8.7 \\
$\mathrm{~A} 10$ & 6.4 & 5.8 & 19.4 & 11.1 & 11.9 & 13.4 & 13.4 & 11.5 & 3.9 & 3.2 \\
$\mathrm{~A} 11$ & 1.2 & 21.8 & 15.6 & 41.3 & 12.8 & 3.6 & 3.1 & 0.0 & 0.3 & 0.3 \\
$\mathrm{~A} 12$ & 11.1 & 8.1 & 10.6 & 6.1 & 32.2 & 8.0 & 18.0 & 3.0 & 2.3 & 0.4 \\
\hline
\end{tabular}

\subsection{Terpene Lactones}

Ginkgo biloba L. contains mainly two active components; the terpene lactones and the ginkgo flavon glycosides, which together have been proven to be responsible for the polyvalent activities of Ginkgo biloba L. containing preparations. The nine commercial samples were analyzed by the developed UPLC/MS-MS method to quantify the levels of terpene lactones in these samples. Figure 2 shows typical chromatograms for the analysis of 5 of terpene lactone standards using the UPLC/MS-MS method under optimized instrument conditions.

According to the analysis results, the B4 coded sample provided the highest free form terpene lactone content $(16.3 \mathrm{mg})$ for daily intake. The samples with the codes of B6 and B7 provided a higher amount of terpene lactone than other products and followed the B4 coded sample in terms of the total quantity. Only two of the analyzed products (B2, B6) were bee product mixtures. Among these products, the B6 coded sample gave the second-highest daily intake value among the analyzed products in terms of terpene lactone values. The results in Table 7 are shown that daily intake amounts of terpene lactones $(\mathrm{mg})$ were ranged between $16.30 \mathrm{mg}$ and $0.10 \mathrm{mg}$. While the ginkgolide B and C are found in all samples, the ginkgolide A was not quantified at only B2 coded sample. Ginkgolide $\mathrm{J}$ and the bilobalide have not been observed in B1, B2 and B3 coded samples. Ginkgolide J was also not quantified in the sample of B6. Analysis results of the investigation for the diversity of terpene lactones in the products are given in Table 8 The homogeneous distribution could only be detected in only a few samples, similar to the results acquired at P. ginseng analyzes. Samples coded B4, B5, B7, B8, B9 became prominent compared to other samples due to the reason that they contain all analyzed terpene lactone parameters and contain four terpene lactone species except ginkgolide $J$ at high and similar percentages. 
Figure 2. The chromatograms of terpene lactones standard solution analysis at $0.5 \mu \mathrm{g} / \mathrm{ml}$ concentration.

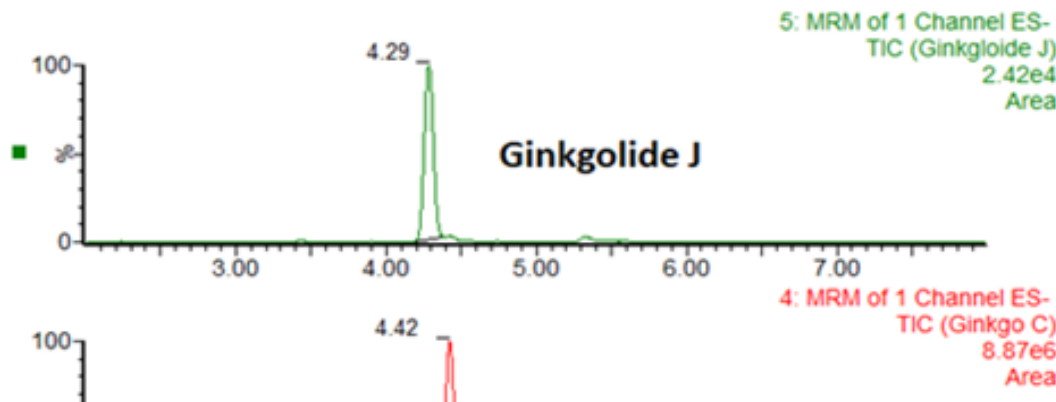

Ginkgolide C
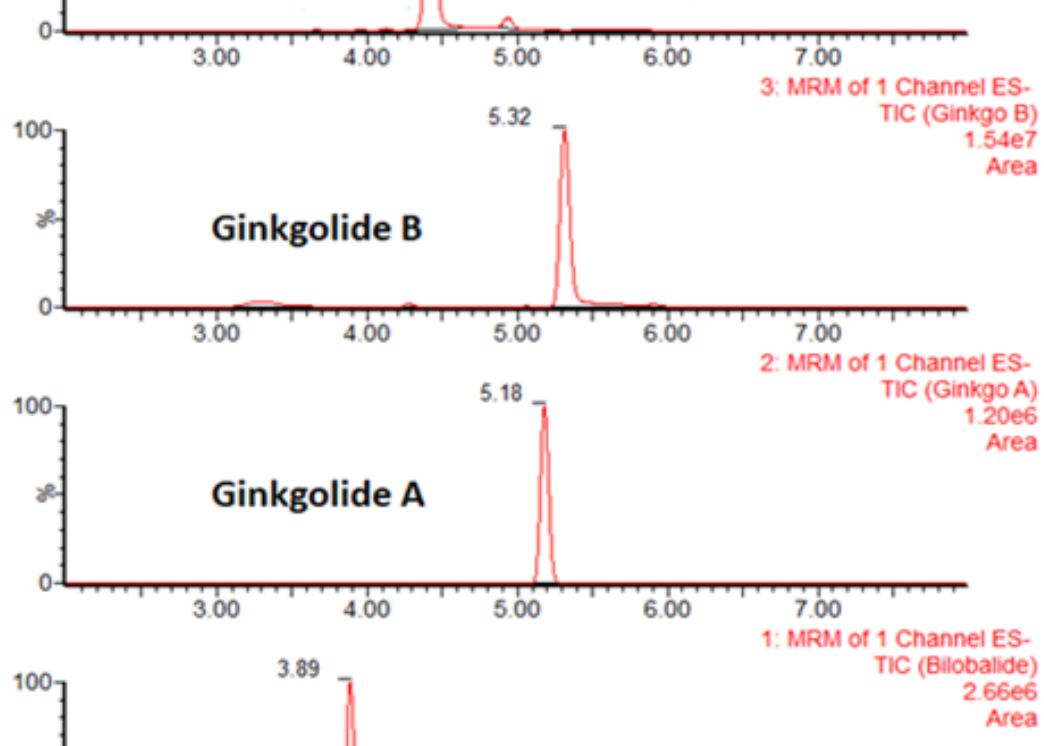

Bilobalide

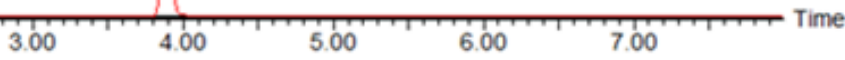


Table 7. Terpene lactone content analysis results of the Ginkgo biloba L. containing samples.

\begin{tabular}{|c|c|c|c|c|c|c|}
\hline Samples & Label claim & Serving size & $\begin{array}{l}\text { Total Terpene Lactone content of } \\
\text { the product }(\mathrm{w} / \mathrm{w} \%) \\
(\mathrm{mg} / 100 \mathrm{mg})\end{array}$ & $\begin{array}{l}\text { Terpene Lactone } \\
\text { content at per } \\
\text { serving }(\mathrm{mg})\end{array}$ & $\begin{array}{l}\text { Recommended daily } \\
\text { serving amount }\end{array}$ & $\begin{array}{l}\text { Quantified daily intake } \\
\text { amounts of Terpene } \\
\text { Lactones (mg) }\end{array}$ \\
\hline B1 & $90 \mathrm{mg}$ of ginkgo leaf extract & 1 tablet $(150 \mathrm{mg})$ & 0.07 & 0.10 & 1 tablet & 0.10 \\
\hline $\mathrm{B} 2^{*}$ & - & $7 \mathrm{~g}$ & 0.02 & 1.05 & $7 \mathrm{~g} \times 3$ & 3.15 \\
\hline B3 & - & $7 \mathrm{~g}$ & 0.03 & 2.10 & $7 \mathrm{~g} \times 3$ & 6.30 \\
\hline B4 & $\begin{array}{c}7.05 \mathrm{mg} \text { of terpene lactone }+117,60 \\
\mathrm{mg} \text { ginkgo leaf extract }\end{array}$ & 1 tablet $(722 \mathrm{mg})$ & 1.13 & 8.16 & 2 tablet & 16.30 \\
\hline B5 & $\begin{array}{c}375 \mathrm{mg} \text { of Ginkgo biloba } \mathrm{L} \text {. leaf } \\
\text { extract }\end{array}$ & 1 tablet $(510 \mathrm{mg})$ & 0.27 & 1.35 & 2 tablet & 2.70 \\
\hline $\mathrm{B} 6^{*}$ & $3.95 \mathrm{mg}$ of terpene lactone & $7 \mathrm{~g}$ & 0.06 & 3.85 & $7 \mathrm{~g} \times 3$ & 11.55 \\
\hline B7 & $\begin{array}{l}5 \mathrm{mg} \text { of terpene lactone }+90 \mathrm{mg} \\
\text { standardized ginkgo extract leaf }\end{array}$ & 1 tablet (316 mg) & 1.74 & 5.48 & 2 tablet & 10.96 \\
\hline B8 & $\begin{array}{c}7.2 \mathrm{mg} \text { of terpene lactone }+120 \mathrm{mg} \\
\text { of ginkgo extract }\end{array}$ & 1 tablet $(870 \mathrm{mg})$ & 1.10 & 9.57 & 1 tablet & 9.57 \\
\hline B9 & $\begin{array}{c}120 \mathrm{mg} \text { of extract ( } 7.2 \mathrm{mg} \text { of terpene } \\
\text { lactone) }\end{array}$ & 1 tablet $(350 \mathrm{mg})$ & 1.92 & 6.70 & 1 tablet & 6.70 \\
\hline
\end{tabular}

\footnotetext{
* Samples are in form of mixtures with apitherapy products.
} 
Table 8. Analysis results of terpene lactone diversities.

\begin{tabular}{cccccc}
\hline \multicolumn{5}{c}{ Terpene lactones by percentage $(\mathrm{w} / \mathrm{w} \%)$} \\
\hline Samples & Bilobalide & Ginkgolide A & Ginkgolide B & Ginkgolide C & Ginkgolide J \\
\hline B1 & 0.0 & 15.4 & 46.2 & 38.5 & 0.0 \\
B2 & 0.0 & 0.0 & 66.7 & 33.3 & 0.0 \\
B3 & 0.0 & 16.7 & 50.0 & 33.3 & 0.0 \\
B4 & 21.7 & 20.8 & 17.3 & 31.4 & 8.8 \\
B5 & 24.5 & 11.3 & 26.4 & 28.3 & 9.4 \\
B6 & 27.3 & 18.2 & 27.3 & 27.3 & 0.0 \\
B7 & 34.9 & 22.2 & 16.1 & 19.9 & 6.9 \\
B8 & 36.8 & 18.2 & 14.1 & 24.1 & 6.8 \\
B9 & 36.6 & 20.4 & 14.4 & 20.9 & 7.8 \\
\hline
\end{tabular}

\subsection{Phenolic profiles and antioxidant capacities}

Previous analysis results have been shown that the forms of P. ginseng and Ginkgo biloba L. extracts mixed with apitherapy products have equivalent concentrations of the active ingredient (ginsenosides and terpene lactones) compared to non-mixed products on the market. The concentration of total and individual phenolic contents is given in Table 9. It can be observed that the content of the 43 components varies greatly among the different samples. Quercetin, rutin hydrate, CAPE, kaempferol, galangin, chrysin, gallic acid, pinocembrin, and isorhamnetin were monitored as predominant phenolic substances as a result of the phenolic profiling analysis. As shown in Figure 3, all these 43 components are analyzed in about $35 \mathrm{~min}$. using novel UPLC/MS-MS method. Figure 4 shows the comparison of the phenolic contents of the bee product supplemented $P$. ginseng samples (A1, A2, A3, A4, A5, A8, A11, and A12) and pure $P$. ginseng extracts (A6, A7, A9, and A10). 
Figure 3. The chromatograms of phenolic profiling analysis at concentration of $0.5 \mu \mathrm{g} / \mathrm{ml}$ standard solution.

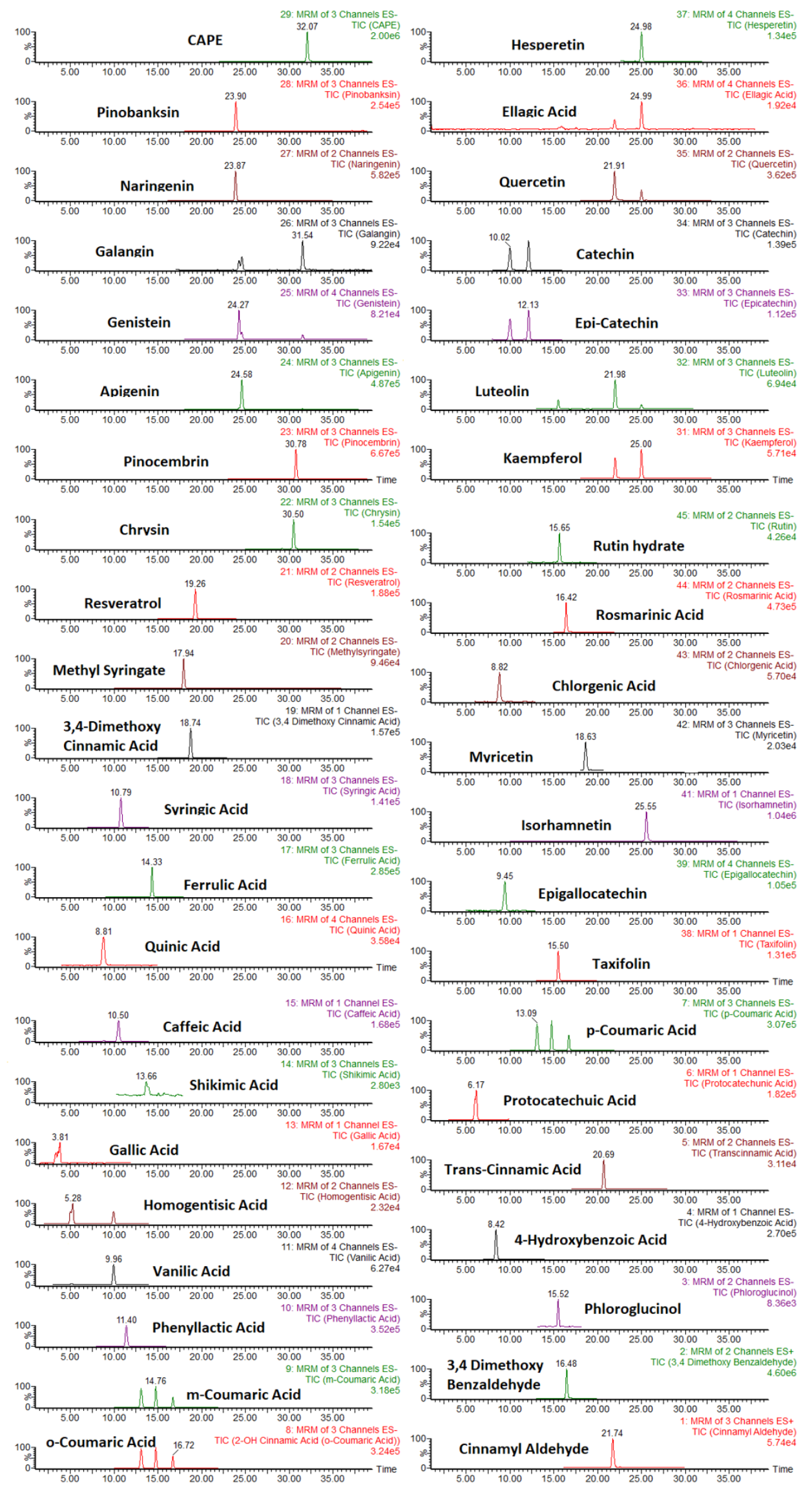


Figure 4. Comparison of selected phenolic compound contents in P.ginseng samples with and without bee product.

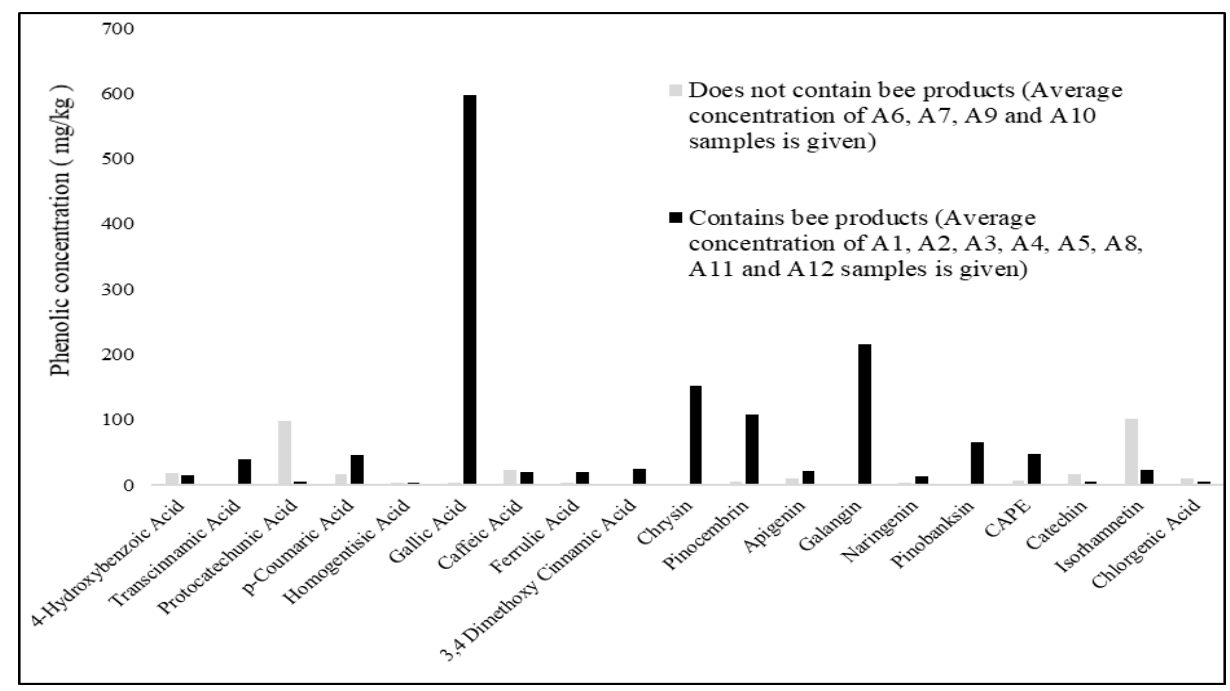

The same effect can also be seen in Figure 5 representing the Ginkgo biloba L. products containing bee products and comparison with those of not containing.

Figure 5. Contribution of bee product supplements in Ginkgo biloba L. extracts to phenolic diversity.

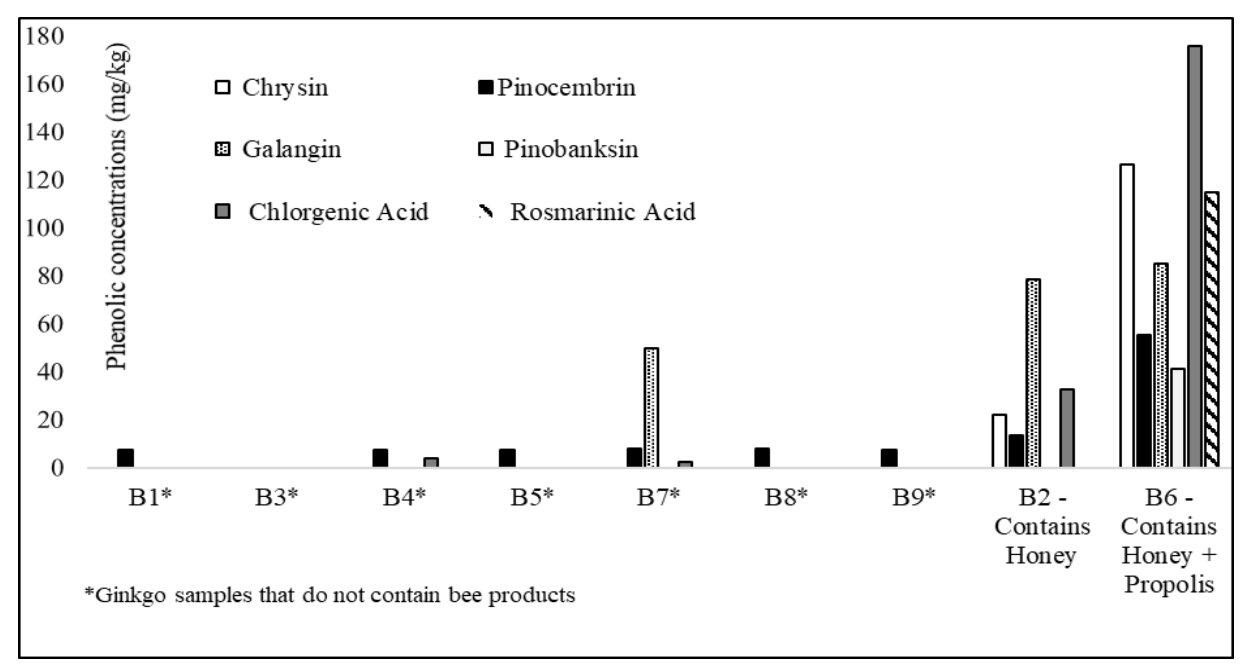

Table $9 \mathrm{a}$ and Table $9 \mathrm{~b}$ also show the antioxidant activity results of the samples which were between $12 \%$ and $23 \%$. According to these results, Ginkgo biloba L. and P. ginseng extracts supported by bee products such as pollen, propolis, and honey also provide high antioxidant capacity besides their phenolic diversities. Getting systematic knowledge about phenolic compounds and mostly flavonoids in the natural products are highly important for the phytotherapy product development strategies but also for the assessment of the therapeutic effects. Therefore, we thought, performing phenolic profiling investigations instead of total phenolic content analysis is much amenable for the samples. It was found that 4hydroxybenzoic acid, transcinnamic acid, protocatechuic acid, p-Coumaric acid, homogentisic acid, gallic acid, caffeic acid, ferulic acid, 3,4-dimethoxy cinnamic acid, chrysin, pinocembrin, apigenin, galangin, naringenin, pinobanksin, CAPE, catechin, quercetin, isorhamnetin, chlorogenic acid, rosmarinic acid, and methylsyringate were the abundant phenolic derivates, which especially arises with the addition of bee products. 
Int. J. Sec. Metabolite, Vol. 8, No. 2, (2021) pp. 70-93

Table 9a. Total antioxidant activities and the phenolic profiles of the $P$. ginseng products.

\begin{tabular}{|c|c|c|c|c|c|c|c|c|c|c|c|c|}
\hline \multirow{2}{*}{ Analyte } & \multicolumn{12}{|c|}{ Sample Results (mg/kg) } \\
\hline & A1 & $\mathrm{A} 2$ & A3 & A4 & A5 & A6 & A7 & $\mathrm{A} 8$ & A9 & A10 & A11 & A12 \\
\hline Cinnamyl Aldehyde & 0.1 & - & - & 1.2 & - & 1.0 & - & - & - & - & 1.7 & - \\
\hline 3,4 Dimethoxy Benzaldehyde & - & - & - & - & - & - & - & - & - & 71.9 & - & - \\
\hline Phloroglucinol & - & - & - & - & - & - & - & - & - & - & - & - \\
\hline 4-Hydroxybenzoic Acid & 1.5 & 11.6 & 14.1 & 69.4 & - & 70.9 & - & 2.7 & - & - & 16.1 & 5.3 \\
\hline Transcinnamic Acid & 13.6 & 28.4 & 89.6 & 161.3 & 0.9 & 0.2 & - & 6.7 & 2.1 & - & 8.0 & 5.2 \\
\hline p-Coumaric Acid & - & 19.8 & 342.1 & 2.9 & - & 68.8 & - & - & - & - & - & 6.5 \\
\hline 2-OH Coumaric Acid & - & - & - & - & - & - & - & - & - & - & - & - \\
\hline m-Coumaric Acid & - & - & - & - & - & - & - & - & - & - & - & - \\
\hline Phenyllactic Acid & - & 2.0 & - & - & - & 18.8 & - & 0.5 & - & - & - & 0.9 \\
\hline Vanilic Acid & - & - & - & - & - & 91.8 & - & - & - & - & - & - \\
\hline Homogentisic Acid & - & 4.1 & 1.9 & 7.3 & 0.7 & - & 0.8 & 3.1 & 7.4 & 3.5 & 0.7 & 2.3 \\
\hline Gallic Acid & 207.1 & 3869.1 & - & 491.7 & - & - & 16.9 & - & - & - & - & 209.8 \\
\hline Shikimic Acid & - & - & - & - & - & - & - & - & - & - & - & - \\
\hline Caffeic Acid & 2.2 & - & 109.7 & 13.9 & - & 89.4 & - & - & - & - & 1.0 & 37.2 \\
\hline Quinic Acid & - & - & - & - & - & - & - & - & - & - & - & - \\
\hline Ferrulic Acid & 0.6 & 29.1 & 117.8 & 2.1 & - & 14.9 & - & - & - & - & - & 4.3 \\
\hline Syringic Acid & 0.2 & 9.3 & - & 0.3 & 1.4 & 33.7 & - & 0.4 & 0.6 & - & - & 0.2 \\
\hline 3,4 Dimethoxycinnamic Acid & - & - & 31.9 & 155.1 & - & - & - & 6.2 & - & - & - & 8.3 \\
\hline Methylsyringate & - & - & - & - & - & - & - & 5.7 & - & - & - & - \\
\hline Resveratrol & - & - & - & - & - & - & - & - & - & - & - & - \\
\hline Chrysin & 26.9 & 25.8 & 337.2 & 497.8 & 26.5 & - & - & 157.9 & - & - & 21.9 & 120.8 \\
\hline Pinocembrin & 10.5 & 24.5 & 248.9 & 338.9 & 8.5 & 7.4 & 7.7 & 76.1 & 7.5 & - & 13.5 & 140.7 \\
\hline Pinobanksin & - & - & 261.4 & 127.0 & - & - & - & 54.5 & - & - & - & 80.3 \\
\hline
\end{tabular}


Table 9a. Continues.

\begin{tabular}{|c|c|c|c|c|c|c|c|c|c|c|c|c|}
\hline CAPE & 12.4 & 10.0 & 164.0 & 50.5 & 11.1 & 8.1 & 7.7 & 9.2 & 7.6 & 7.2 & 8.0 & 120.6 \\
\hline Kaempferol & - & - & - & - & - & 477.7 & - & - & - & 40.3 & - & - \\
\hline Apigenin & - & - & 48.8 & 51.7 & - & 40.6 & - & 24.3 & - & - & 17.7 & 28.9 \\
\hline Galangin & 48.0 & 199.2 & 287.1 & 816.2 & 32.4 & - & - & 176.2 & - & - & 78.3 & 85.8 \\
\hline Naringenin & - & 1.5 & 46.6 & 32.6 & - & 12.5 & - & 8.1 & - & - & 0.1 & 13.0 \\
\hline Luteolin & - & - & - & - & - & - & - & - & - & - & - & - \\
\hline Epicatechin & - & - & - & - & - & 11.6 & - & 5.3 & - & - & - & - \\
\hline Catechin & - & - & - & 11.6 & - & 68.8 & - & 28.8 & - & - & - & - \\
\hline Quercetin & 27.2 & 24.9 & 25.6 & 52.5 & - & 3897.7 & 29.4 & 26.2 & 16.9 & 188.6 & 33.0 & 80.0 \\
\hline Ellagic Acid & - & - & - & - & - & - & - & - & - & - & - & - \\
\hline Hesperetin & - & - & 2.1 & 4.1 & 2.6 & - & 3.2 & 2.9 & 3.0 & 3.3 & 2.0 & 2.8 \\
\hline Taxifolin & - & - & - & - & - & - & - & - & - & - & - & - \\
\hline Isorhamnetin & 17.1 & 15.1 & 33.1 & 46.9 & 12.6 & 368.3 & 12.6 & 19.8 & 12.5 & 15.7 & 17.1 & 18.4 \\
\hline Myricetin & - & - & - & - & - & - & - & - & - & - & - & - \\
\hline Chlorgenic Acid & 4.9 & - & - & - & - & 3.8 & - & - & 35.3 & - & 32.6 & 1.8 \\
\hline Rosmarinic Acid & - & - & - & - & - & - & - & - & - & - & - & - \\
\hline Rutin & - & - & - & 2.0 & - & 1609.3 & - & 0.4 & - & - & 33.4 & - \\
\hline Total Phenolics (mg/g) & 0.4 & 4.3 & 2.2 & 3.0 & 0.1 & 7.8 & 0.1 & 0.6 & 0.1 & 0.3 & 0.3 & 1.0 \\
\hline Total Antioxidant Activity (\%) & 90.5 & 83.9 & 87.7 & 89.1 & 13.0 & 85.2 & 20.7 & 61.1 & 30.8 & 38.4 & 63.7 & 92.0 \\
\hline
\end{tabular}


Table 9b. Total antioxidant activities and the phenolic profiles of Ginkgo biloba L. products.

\begin{tabular}{|c|c|c|c|c|c|c|c|c|c|}
\hline \multirow{2}{*}{ Analyte } & \multicolumn{9}{|c|}{ Sample Results (mg/kg) } \\
\hline & $\mathrm{B} 1$ & $\mathrm{~B} 2$ & B3 & B4 & B5 & B6 & B7 & B8 & B9 \\
\hline Cinnamyl Aldehyde & - & 1.7 & - & 1.0 & 1.3 & - & 2.2 & - & - \\
\hline 3,4 Dimethoxy Benzaldehyde & - & - & - & - & - & - & - & - & - \\
\hline Phloroglucinol & - & - & - & - & - & - & - & - & - \\
\hline 4-Hydroxybenzoic Acid & 49.2 & 16.1 & 1.3 & 70.9 & 142.8 & 5.9 & 249.8 & 159.3 & 306.5 \\
\hline Transcinnamic Acid & - & 8.0 & - & 0.2 & - & 14.0 & 11.0 & - & 15.6 \\
\hline Protocatechunic Acid & 35.4 & 2.8 & 2.6 & 392.0 & 158.5 & 21.7 & 901.2 & 94.2 & 914.4 \\
\hline p-Coumaric Acid & 7.6 & - & - & 68.8 & 73.9 & - & 178.1 & 73.5 & 314.2 \\
\hline 2-OH Coumaric Acid & - & - & - & - & - & - & - & - & - \\
\hline m-Coumaric Acid & - & - & - & - & - & - & - & - & - \\
\hline Phenyllactic Acid & - & - & - & 18.8 & - & 1.7 & - & - & - \\
\hline Vanilic Acid & - & - & - & 91.8 & 36.4 & - & 125.5 & 55.4 & 174.8 \\
\hline Homogentisic Acid & 3.3 & 0.7 & 1.6 & - & - & 3.5 & 4.9 & 7.6 & 23.1 \\
\hline Gallic Acid & - & - & - & - & - & - & - & - & 128.8 \\
\hline Shikimic Acid & - & - & - & - & - & - & - & - & - \\
\hline Caffeic Acid & 0.4 & 1.0 & - & 89.4 & 5.4 & 3.8 & 10.2 & 12.6 & 16.4 \\
\hline Quinic Acid & - & - & - & - & - & - & - & - & - \\
\hline Ferrulic Acid & - & - & - & 14.9 & - & - & 19.3 & 4.4 & 34.8 \\
\hline Syringic Acid & 4.8 & - & 0.2 & 33.7 & 11.2 & 2.7 & 33.0 & 9.8 & 42.1 \\
\hline 3,4 Dimethoxycinnamic Acid & - & - & - & - & - & - & - & - & - \\
\hline Methylsyringate & - & - & - & - & - & 2.4 & - & - & - \\
\hline Resveratrol & - & - & - & - & - & - & - & - & - \\
\hline Chrysin & - & 21.9 & - & - & - & 126.2 & - & - & - \\
\hline Pinocembrin & 7.5 & 13.5 & - & 7.4 & 7.4 & 55.0 & 7.7 & 7.7 & 7.5 \\
\hline Pinobanksin & - & - & - & - & - & 41.0 & - & - & - \\
\hline CAPE & 9.4 & 8.0 & 8.3 & 8.1 & 7.6 & 9.7 & 7.4 & 7.7 & 7.3 \\
\hline Kaempferol & - & - & - & 477.7 & - & 109.1 & 5009.5 & 640.8 & 5432.2 \\
\hline Apigenin & 21.1 & 17.7 & - & 40.6 & 36.5 & 21.7 & 85.9 & 52.0 & 98.4 \\
\hline
\end{tabular}


Akyildiz, et al.,

Table 9b. Continues

\begin{tabular}{|c|c|c|c|c|c|c|c|c|c|}
\hline Genistein & - & - & - & 461.1 & - & - & 114.3 & - & 161.5 \\
\hline Galangin & - & 78.3 & - & - & - & 84.9 & 49.7 & - & - \\
\hline Naringenin & 3.2 & 0.1 & - & 12.5 & 21.6 & 10.3 & 40.6 & 23.2 & 36.6 \\
\hline Luteolin & - & - & - & - & - & - & 54.8 & - & 44.3 \\
\hline Epicatechin & - & - & - & 11.6 & - & - & - & - & - \\
\hline Catechin & - & - & - & 68.8 & 33.6 & 8.3 & 19.3 & 10.9 & 24.6 \\
\hline Quercetin & 19.7 & 33.0 & - & 3897.7 & 178.5 & 185.4 & 4483.7 & 913.5 & 5065.9 \\
\hline Ellagic Acid & - & - & - & - & - & - & - & - & - \\
\hline Hesperetin & - & 2.0 & - & - & 2.8 & - & 117.6 & 10.2 & 105.8 \\
\hline Taxifolin & - & - & - & - & 11.5 & - & 2.1 & 4.6 & 14.4 \\
\hline Epigallocatechin & 0.8 & 1.1 & 1.5 & 63.0 & 9.0 & 1.3 & 2.8 & 2.1 & 1.5 \\
\hline Isorhamnetin & 15.6 & 17.1 & 12.5 & 368.3 & 20.0 & 24.0 & 706.8 & 249.7 & 810.3 \\
\hline Myricetin & - & - & - & - & - & - & - & - & - \\
\hline Chlorgenic Acid & - & 32.6 & - & 3.8 & - & 175.8 & 2.4 & - & - \\
\hline Rosmarinic Acid & - & - & - & - & - & 114.9 & - & - & - \\
\hline Rutin & 102.6 & 33.4 & - & 1609.3 & 347.5 & 14.6 & 1859.5 & 2004.5 & 1894.4 \\
\hline Total Phenolics (mg/g) & 0.3 & 0.3 & 0.1 & 7.8 & 1.1 & 1.0 & 14.1 & 4.3 & 15.7 \\
\hline Total Antioxidant Activity (\%) & 72.5 & 63.7 & 20.3 & 85.2 & 85.3 & 91.1 & 71.2 & 83.5 & 74.1 \\
\hline
\end{tabular}


According to results, propolis and pollen were mainly contributed to the phenolic profiles more than the royal jelly and honey supports.

Previous research based on the identification of the secondary metabolites of $P$. ginseng and Ginkgo biloba L. supplements includes tedious sample preparation steps, insensitive detection methods, and identifications based on passive detector acquisitions such as refractive index detection, UV detection, and so forth (Wang \& Ju, 2000; Li et al., 2005; Tang et al., 2010). UPLC-ESI-MS/MS and developed methods enabled to identify and quantify the compounds of interest more accurately, specifically owing to their unique three-dimensional data (retention time, abundance, and mass to charge ratio). In addition to this, comprehensive phenolic profiling analysis of these herbal extracts was not achieved until this novel method was implemented. In this study, ultrasound-assisted solid-liquid extracts of the samples were analyzed directly in the UPLC-ESI-MS/MS system for three different phytochemical classes (terpene lactones, ginsenosides, and phenolics) without any additional purification, concentration, or derivatization. By using a single extract obtained from these samples, antioxidant and active ingredient measurements could be made simultaneously. In this way, the preliminary process has been simplified.

\section{CONCLUSION}

The active ingredient contents of $P$. ginseng and Ginkgo biloba L. supplements available in the market and the forms that supplemented with bee products were enlightened. Contents of the supplied samples were evaluated in terms of potential phytotherapy efficacy by means of research carried out using novel developed analytical methods. Within the scope of the study, phenolic substances analysis method including 43 compounds was developed using UltraPerformance Liquid Chromatography, electrospray ionization (ESI) Tandem Mass Spectrometry (UPLC-ESI-MS/MS) system. Thus, sensitive and high-resolution profiling study was achieved. In addition, analytical methods were developed based on MS detection for the monitoring of ginkgolide, bilobalide, and ginsenoside active compounds. At our investigation, it has been determined that $P$. ginseng and Ginkgo biloba L. supplements available in the market predominantly contain sufficient amounts of active ingredients, as well as the analyzed amounts and compositions, were variable. The novel and practical analytical methods proved that the health benefits of the aforementioned products will increase owing to the fact that an increase in the phytochemical molecule diversity and antioxidant capacities if the content of the raw extract is supported with alternative apitherapy products.

\section{Acknowledgments}

Analysis within the scope of the study was carried out at Balparmak R\&D Center. The authors of this study would like to thank all the Balparmak R\&D Center team and the members of the board of directors who provided the necessary facilities for the study.

\section{Declaration of Conflicting Interests and Ethics}

The authors declare no conflict of interest. This research study complies with research and publishing ethics. The scientific and legal responsibility for manuscripts published in IJSM belongs to the authors.

\section{Authorship Contribution Statement}

Ismail Emir Akyildiz: Writing-Original draft preparation, Methodology, Conceptualization. Sinem Raday: Writing-Reviewing and Editing. Ozge Erdem: Resources. Sezer Acar: Investigation. Ilknur Coskun: Supervision. Emel Damarli: Project administration. 


\section{Orcid}

Ismail Emir Akyıldız (iD https://orcid.org/0000-0003-0644-0405

Sinem Raday (iD https://orcid.org/0000-0002-4683-070X

Ozge Erdem (iD https://orcid.org/0000-0001-7883-9250

Sezer Acar (D) https://orcid.org/0000-0001-9883-4385

Ilknur Coskun (iD https://orcid.org/0000-0002-6796-9065

Emel Damarli id https://orcid.org/0000-0003-1082-2430

\section{REFERENCES}

Chan, T., But, P., Cheng, S., Kwok, I., Lau, F., \& Xu, H. (2000). Differentiation and authentication of Panax ginseng, Panax quinquefolius, and ginseng products by using HPLC/MS. Analytical Chemistry, 72(6), 1281-1287.

Chang, J., \& Chang, M. (1997). Medicinal uses of Ginkgo biloba. Todays Therapeutic Trends, $15,63-74$

Court, W. A., Hendel, J. G., \& Elmi, J. (1996). Reversed-phase high-performance liquid chromatography determination of ginsenosides of Panax quinquefolium. Journal of Chromatography a, 755(1), 11-17.

Czigle, S., Héthelyi B, É., Háznagy-Radnai, E., Máthé, I., \& Tóth, J. (2019). Analysis of Volatile Constituents of Ginkgo Leaf. Natural Product Communications, 14(6), $1934578 X 19857900$.

de Jager, L. S., Perfetti, G. A., \& Diachenko, G. W. (2006). Analysis of ginkgolides and bilobalide in food products using LC-APCI-MS. Journal of Pharmaceutical and Biomedical Analysis, 41(5), 1552-1559.

DeFeudis, F. V. (1998). Ginkgo biloba extract (EGb 761): from chemistry to the clinic (Vol. 25). Ullstein Medical Wiesbaden.

Diamond, B. J., Shiflett, S. C., Feiwel, N., Matheis, R. J., Noskin, O., Richards, J. A., \& Schoenberger, N. E. (2000). Ginkgo biloba extract: mechanisms and clinical indications. Archives of Physical Medicine and Rehabilitation, 81(5), 668-678.

Dou, D.-Q., Hou, W.-B., \& Chen, Y.-J. (1998). Studies of the characteristic constituents of Chinese ginseng and American ginseng. Planta Medica, 64(06), 585-586.

Dubber, M.-J., \& Kanfer, I. (2006). Determination of terpene trilactones in Ginkgo biloba solid oral dosage forms using HPLC with evaporative light scattering detection. Journal of Pharmaceutical and Biomedical Analysis, 41(1), 135-140.

Gui, Y., \& Ryu, G.-H. (2014). Effects of extrusion cooking on physicochemical properties of white and red ginseng (powder). Journal of Ginseng Research, 38(2), 146-153.

Hong, H.-D., Sim, E. M., Kim, K., Rho, J., Rhee, Y. K., \& Cho, C.-W. (2009). Comparison of preparation methods for the quantification of ginsenosides in raw Korean ginseng. Food Science and Biotechnology, 18(2), 565-569.

Jacobs, B. P., \& Browner, W. S. (2000). Ginkgo biloba: A living fossil. American journal of Medicine, 108(4), 341-342.

Kaur, P., Chaudhary, A., \& Singh, B. (2009). Optimization of extraction technique and validation of developed RP-HPLC-ELSD method for determination of terpene trilactones in Ginkgo biloba leaves. Journal of Pharmaceutical and Biomedical Analysis, 50(5), 10601064

Kiefer, M. (2004). Review about Ginkgo biloba special extract EGb 761 (Ginkgo). Current Pharmaceutical Design, 10(3), 261.

Kim, J.-H. (2018). Pharmacological and medical applications of Panax ginseng and ginsenosides: a review for use in cardiovascular diseases. Journal of Ginseng Research, 42(3), 264-269. 
Kim, J. H., Yi, Y.-S., Kim, M.-Y., \& Cho, J. Y. (2017). Role of ginsenosides, the main active components of Panax ginseng, in inflammatory responses and diseases. Journal of Ginseng Research, 41(4), 435-443.

Kim, K. H., Lee, D., Lee, H. L., Kim, C.-E., Jung, K., \& Kang, K. S. (2018). Beneficial effects of Panax ginseng for the treatment and prevention of neurodegenerative diseases: past findings and future directions. Journal of Ginseng Research, 42(3), 239-247.

Le Bars, P., Velasco, F., Ferguson, J., Dessain, E., Kieser, M., \& Hoerr, R. (2002). Influence of the severity of cognitive impairment on the effect of the Gnkgo biloba extract EGb 761 in Alzheimer's disease. Neuropsychobiology, 45(1), 19.

Lee, S. M., Bae, B.-S., Park, H.-W., Ahn, N.-G., Cho, B.-G., Cho, Y.-L., \& Kwak, Y.-S. (2015). Characterization of Korean Red Ginseng (Panax ginseng C.A. Meyer): History, preparation method, and chemical composition. Journal of Ginseng Research, 39(4), 384-391.

Li, L., Zhang, J.-1., Sheng, Y.-X., Guo, D.-a., Wang, Q., \& Guo, H.-z. (2005). Simultaneous quantification of six major active saponins of Panax notoginseng by high-performance liquid chromatography-UV method. Journal of Pharmaceutical and Biomedical Analysis, $38(1), 45-51$.

Liu, X.-G., Wu, S.-Q., Li, P., \& Yang, H. (2015). Advancement in the chemical analysis and quality control of flavonoid in Ginkgo biloba. Journal of Pharmaceutical and Biomedical Analysis, 113, 212-225.

Mahadevan, S., \& Park, Y. (2008). Multifaceted therapeutic benefits of Ginkgo biloba L.: chemistry, efficacy, safety, and uses. Journal of food Science, 73(1), R14-R19.

Nash, K. M., \& Shah, Z. A. (2015). Current perspectives on the beneficial role of Ginkgo biloba in neurological and cerebrovascular disorders. Integrative Medicine Insights, 10, IMI. S25054.

Pietri, S., Maurelli, E., Drieu, K., \& Culcasi, M. (1997). Cardioprotective and Anti-oxidant Effects of the Terpenoid Constituents of Ginkgo biloba Extract (EGb 761). Journal of Molecular and Cellular Cardiology, 29(2), 733-742.

Popovich, D. G., Yeo, C.-R., \& Zhang, W. (2012). Ginsenosides derived from Asian (Panax ginseng), American ginseng (Panax quinquefolius) and potential cytoactivity. International Journal of Biomedical and Pharmaceutical Sciences, 6(1), 56-62.

Qi, L.-W., Wang, C.-Z., \& Yuan, C.-S. (2011). Ginsenosides from American ginseng: chemical and pharmacological diversity. Phytochemistry, 72(8), 689-699.

Sayadi, L., Missaoui, I., Jamoussi, B., \& Abderraba, A. (2010). Development and Validation of a Gas Chromatographic Method for Identification and Quantification of Terpene Trilactones in Ginkgo biloba L. Extract and Pharmaceutical Preparations. The Open Chemical and Biomedical Methods Journal, 3, 18-24.

Shin, B.-K., Kwon, S. W., \& Park, J. H. (2015). Chemical diversity of ginseng saponins from Panax ginseng. Journal of ginseng research, 39(4), 287-298.

Shin, J.-Y., Choi, E.-H., \& Wee, J.-J. (2001a). The difference of ginsenoside compositions according to the conditions of extraction and fractionation of crude ginseng saponins. Korean Journal of Food Science and Technology, 33(3), 282-287.

Shin, J.-Y., Choi, E.-H., \& Wee, J.-J. (2001b). New methods for separation of crude ginseng saponins. Korean Journal of Food Science and Technology, 33(2), 166-172.

Smith, J., \& Luo, Y. (2004). Studies on molecular mechanisms of Ginkgo biloba extract. Applied microbiology and biotechnology, 64(4), 465-472.

Stiker, O., Meier, B., \& Hasler, A. (2000). The analysis of ginkgo flavonoids, in van Beek TA (ed), Ginkgo biloba. In: Harwood, Amsterdam.

Tang, D., Yang, D., Tang, A., Gao, Y., Jiang, X., Mou, J., \& Yin, X. (2010). Simultaneous chemical fingerprint and quantitative analysis of Ginkgo biloba extract by HPLC-DAD. Analytical and Bioanalytical Chemistry, 396(8), 3087-3095. 
van Beek, T. A. (2002). Chemical analysis of Ginkgo biloba leaves and extracts. Journal of Chromatography a, 967(1), 21-55.

van Beek, T. A., \& Montoro, P. (2009). Chemical analysis and quality control of Ginkgo biloba leaves, extracts, and phytopharmaceuticals. Journal of Chromatography a, 1216(11), 20022032.

Wang, H., \& Ju, X. (2000). Rapid analysis of terpene lactones in extract of Ginkgo biloba L. by high performance liquid chromatography. Se pu=Chinese journal of chromatography, 18(5), 394.

Wang, Y., Liu, Y., Wu, Q., Yao, X., \& Cheng, Z. (2017). Rapid and sensitive determination of major active ingredients and toxic components in Ginkgo biloba leaves extract (EGb 761) by a validated UPLC-MS-MS Method. Journal of Chromatographic Science, 55(4), 459464.

Xia, Y.-G., Song, Y., Liang, J., Guo, X.-D., Yang, B.-Y., \& Kuang, H.-X. (2018). Quality Analysis of American Ginseng Cultivated in Heilongjiang Using UPLC-ESI--MRM-MS with Chemometric Methods. Molecules, 23(9), 2396.

Yang, W.-z., Hu, Y., Wu, W.-y., Ye, M., \& Guo, D.-a. (2014). Saponins in the genus Panax L.(Araliaceae): a systematic review of their chemical diversity. Phytochemistry, 106, 7-24.

Yu, J., Xu, T., Lin, H., Lin, Y., Zhou, J., \& Zhang, Y. (2021). Comprehensive Quality Evaluation of American Ginseng for Different Parts and Abnormal Trait Based on the Major Ginsenoside Contents and Morphological Characteristics. BioMed Research International, 2021 . 